\title{
Oscillating field stimulation promotes recovery from spinal cord injury in rats by regulating the differentiation of endogenous neural stem cells
}

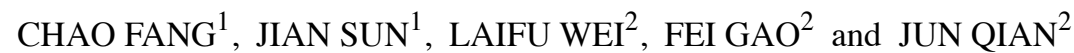 \\ ${ }^{1}$ Department of Orthopedics, The Second Affiliated Hospital of Anhui Medical University, \\ Hefei, Anhui 230601; ${ }^{2}$ Department of Spine Surgery, The First Affiliated Hospital \\ of Anhui Medical University, Hefei, Anhui 230031, P.R. China
}

Received January 8, 2020; Accepted June 26, 2020

DOI: $10.3892 /$ etm.2021.10411

\begin{abstract}
The mammalian spinal cord (SC) has a limited selfrepair capacity and exogenous treatments are yet to produce substantial functional recovery following SC injury (SCI). The SC contains endogenous neural stem cells (NSCs) with multi-lineage differentiation potential and it may be possible to restore function via interventions that promote NSC differentiation following SCI. Oscillating field stimulation (OFS) has been reported to regulate the Wnt signaling pathway, a known modulator of NSC differentiation. However, the effects of OFS on NSC differentiation following SCI and associated functional recovery have not been previously examined. In the current study, the Basso-Beattie-Bresnahan (BBB) score was used to assess locomotion recovery following $\mathrm{SCI}$ in rats and immunofluorescence double-staining was used to examine the regeneration of neurons and oligodendrocytes derived from NSCs. Furthermore, Nissl staining was performed to assess the viability and survival of neurons following SCI, while recovery of the myelin sheath was examined by uranium-lead staining under transmission electron microscopy. OFS delivered via an implanted stimulator enhanced the differentiation of NSCs into neurons and oligodendrocytes and accelerated the regeneration of myelinated axons. Additionally, BBB scores revealed superior locomotion recovery in OFS-treated rats compared with SCI controls. Collectively, these results indicated that OFS may be a feasible strategy to promote SCI recovery by regulating the differentiation of endogenous NSCs.
\end{abstract}

Correspondence to: Dr Jun Qian, Department of Spine Surgery, The First Affiliated Hospital of Anhui Medical University, 218 Jixi Road, Hefei, Anhui 230031, P.R. China

E-mail: qjpaper@sina.cn

Key words: spinal cord injury, oscillating field stimulation, endogenous neural stem cells, differentiation, recovery

\section{Introduction}

Spinal cord injury (SCI) often results in permanent disability, placing a subsequent emotional and financial burden on patients, families and society $(1,2)$. Currently, SCI therapy includes corticosteroidal drugs, relieving excessive spinal cord (SC) pressure and pain and rehabilitation training; however, overcoming the limited self-repair capacity of the $\mathrm{SC}$ has proven challenging $(3,4)$. The regeneration and functional recovery of the SC requires the precisely regulated induction of multiple cellular processes, including regrowth of injured axons, production of new axons, re-establishment of precise synaptic connections and restoration of axonal conduction by electrophysiological recovery and remyelination (5). Thus, the development of strategies for inducing neural regeneration following SCI is a major aim of clinical neuroscience.

The mammalian SC possesses a population of endogenous neural stem cells (NSCs) (6) that can differentiate into neurons, astrocytes and oligodendrocytes in response to specific factors in the SCI microenvironment and form new synaptic connections to re-establish nerve transmission pathways $(7,8)$. In addition to axonal regrowth and repair following SCI, re-establishment of myelination by oligodendrocytes is essential for functional recovery (9). Therefore, repair strategies utilizing NSCs must induce efficient oligodendrocyte differentiation, axonal repair and/or the production of new neurons.

Oscillating field stimulation (OFS) is considered a promising therapeutic strategy for neural regeneration and has demonstrated modest clinical efficacy (10). However, the underlying mechanisms are still unknown, which hinders optimization for broader clinical application. Previous research has shown that electric field stimulation regulates Wnt protein expression and Wnt signaling pathways (11). Wnt signaling regulates NSC differentiation, which indicates that OFS-induced modulation of Wnt signaling may be a potential therapeutic strategy for SCI (12). However, whether OFS can promote neurological recovery following SCI by regulating the differentiation of endogenous NSCs has not been previously reported. 
In the current study, the capacity of OFS to enhance endogenous NSC differentiation and promote functional recovery was examined in a rat model of SCI. The present study aimed to provide a theoretical basis for assessing the clinical value of OFS in the treatment of SCI.

\section{Materials and methods}

Animals. A total of 72 adult female Sprague-Dawley rats (age, $~ 8$ weeks; weight, $220 \pm 10$ g) were purchased from Anhui Medical University. The rats were raised in an animal room under controlled ambient temperature $\left(26^{\circ} \mathrm{C}\right)$ and $60 \%$ relative humidity on a 12-h light/dark cycle with ad libitum access to food and water. The rats were randomly allocated to three groups with 24 rats in each group: Sham controls, SCI alone and SCI + OFS. The Sham group underwent laminectomy without SCI surgery. The SCI and SCI + OFS groups were subjected to laminectomy and experimental SCI. Rats in the SCI group experienced the implantation of the stimulator, though this was not activated so they did not receive OFS, while rats in the SCI + OFS group underwent OFS as the intervention. All surgical procedures and experiments were performed in accordance with the National Institutes of Health Guide for the Care and Use of Laboratory Animals (NIH Publications no. 80-23; revised 1996) (13) and were approved by the Animal Ethics Committee of Anhui Medical University (approval reference no. LLSC20190736).

OFS device. The OFS device was provided by the Beijing Key Laboratory of Bioelectromagnetics, Institute of Electrical Engineering, Chinese Academy of Sciences. The parameters of functionality, implantability and biocompatibility were considered when selecting a device, so as to have the least negative impact on the animals. The device was powered by a 3.0-V primary battery (cat. no. CR1220; Panasonic Corporation) with an output current of $40 \mu \mathrm{A}$ and an electric field strength of $400 \mu \mathrm{V} / \mathrm{mm}$ between positive and negative electrodes. The structure and working mechanism of the OFS device are described in a previous study (14).

Experimental SCI. The rats were anesthetized via intraperitoneal injection of $300 \mathrm{mg} / \mathrm{kg}$ chloral hydrate and then fixed on an operating table. Following hair removal from the back and skin disinfection, a $3-\mathrm{cm}$ longitudinal incision was made at the dorsal midline overlying the lower thoracic $(\mathrm{T})$ vertebrae. The SC at T9-T10 was exposed by blunt dissection of the paravertebral muscles and removal of spinous processes and laminae, while carefully avoiding additional tissue damage. A $10 \mathrm{~g}$ New York University Multicenter Animal Spinal Cord Injury Study impactor was used to model SCI by striking the center of the exposed SC vertically from a height of $3 \mathrm{~cm}(15)$. Furthermore, in the SCI + OFS and SCI groups, OFS device electrodes $(25 \mathrm{~mm} \times 7 \mathrm{~mm})$ were sutured in the intervertebral muscles above and below the injury site and the device was stitched to the back epidermis. In all groups, the wounds were sutured layer-by-layer using 4-0 silk.

Body temperature was maintained at $37^{\circ} \mathrm{C}$ during the operation using a heating lamp. All animals were subcutaneously injected with $10^{5} \mathrm{U} / \mathrm{kg}$ penicillin $\mathrm{G}$ once per day for 3 days post-surgery. No signs of peritonitis were observed in

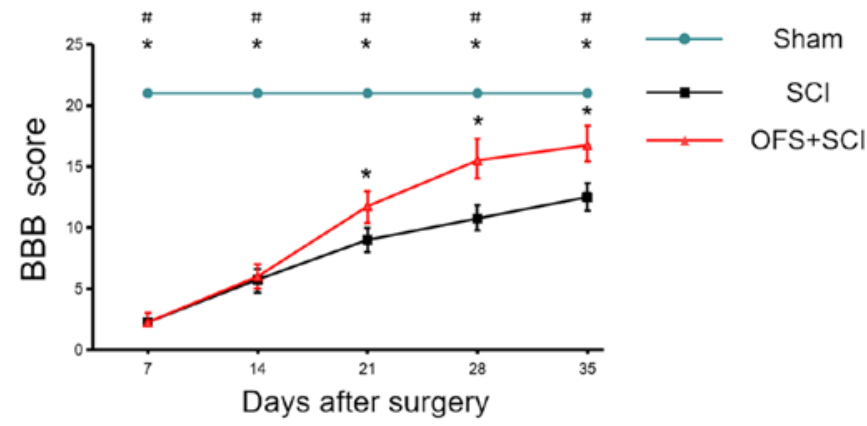

Figure 1. Locomotion recovery was assessed using BBB scores post-surgery. BBB scores were examined and recorded at days 7, 14, 21, 28 and 35 postsurgery. Two-way ANOVA repeated measurement followed by Tukey's post hoc test. ${ }^{*} \mathrm{P}<0.05$ vs. SCI group; ${ }^{~} \mathrm{P}<0.05$ vs. OFS + SCI group. Sham, controls that underwent laminectomy only; SCI, group that underwent laminectomy and spinal cord injury; SCI + OFS, group that underwent laminectomy, spinal cord injury and OFS; BBB, Basso-Beattie-Bresnahan; OFS, Oscillating field stimulation.

any group following surgery. Manual bladder emptying was performed by squeezing of the bladder every $8 \mathrm{~h}$ until rats resumed autonomous voiding.

Behavioral analysis. The Basso-Beattie-Bresnahan (BBB) score is a widely accepted tool for assessing recovery of motor function following SCI (16). Rats were scored multiple times a day at the indicated time points by observing motor function and co-ordination of hind limbs on days 7, 14, 21, 28 and 35 post-surgery. Each rat was evaluated by two independent observers, who were blinded to the animals' group assignment, and the mean BBB scores were used in the final analysis.

Double immunofluorescence (IF) staining and microscopy. From each group, 4 rats were sacrificed on days 3, 7 and 14 post-surgery with excessive pentobarbital sodium anesthesia $(150 \mathrm{mg} / \mathrm{kg})$ and a $1-\mathrm{cm} \mathrm{SC}$ section centered at the injury site was removed. Excised spinal tissue was fixed in $4 \%$ paraformaldehyde at room temperature for $12 \mathrm{~h}$, embedded in paraffin and cut into serial $10-\mu \mathrm{m}$ thick transverse sections. Tissue sections were immersed in EDTA (cat. no. G1206; Servicebio Technology, Co., Ltd.) at $60^{\circ} \mathrm{C}$ for $25 \mathrm{~min}$ to promote antigen repair. After rinsing in PBS, sections were blocked in $5 \%$ bovine serum albumin (BSA; G5001; Servicebio Technology Co., Ltd.) at room temperature for $30 \mathrm{~min}$ and then incubated with primary antibodies against $\beta$-tubulin III (1:200; cat. no. T8578; Sigma-Aldrich; Merck KGaA) and neuronglial antigen 2 (NG2; 1:200; cat. no. ab50009; Abcam) in QuickBlock $^{\mathrm{TM}}$ primary antibody dilution buffer (cat.no. P0262; Beyotime Institute of Biotechnology) at $4^{\circ} \mathrm{C}$ overnight. Each section was washed with PBS three times and incubated with horseradish peroxidase-labeled goat anti-mouse $\operatorname{IgG}$ (1:200; cat. no. GB23301; Servicebio Technology, Co., Ltd.) in QuickBlock secondary antibody dilution buffer for immunofluorescence (cat. no. p0265; Beyotime Institute of Biotechnology) for $1 \mathrm{~h}$ at room temperature. Sections were stained with FITC-conjugated donkey anti-goat IgG (1:100; cat. no. GB22404; Servicebio Technology, Co., Ltd.) in Quickblock secondary antibody dilution buffer at room temperature for $10 \mathrm{~min}$ and then immersed in EDTA and heated in a microwave oven to remove the 
A
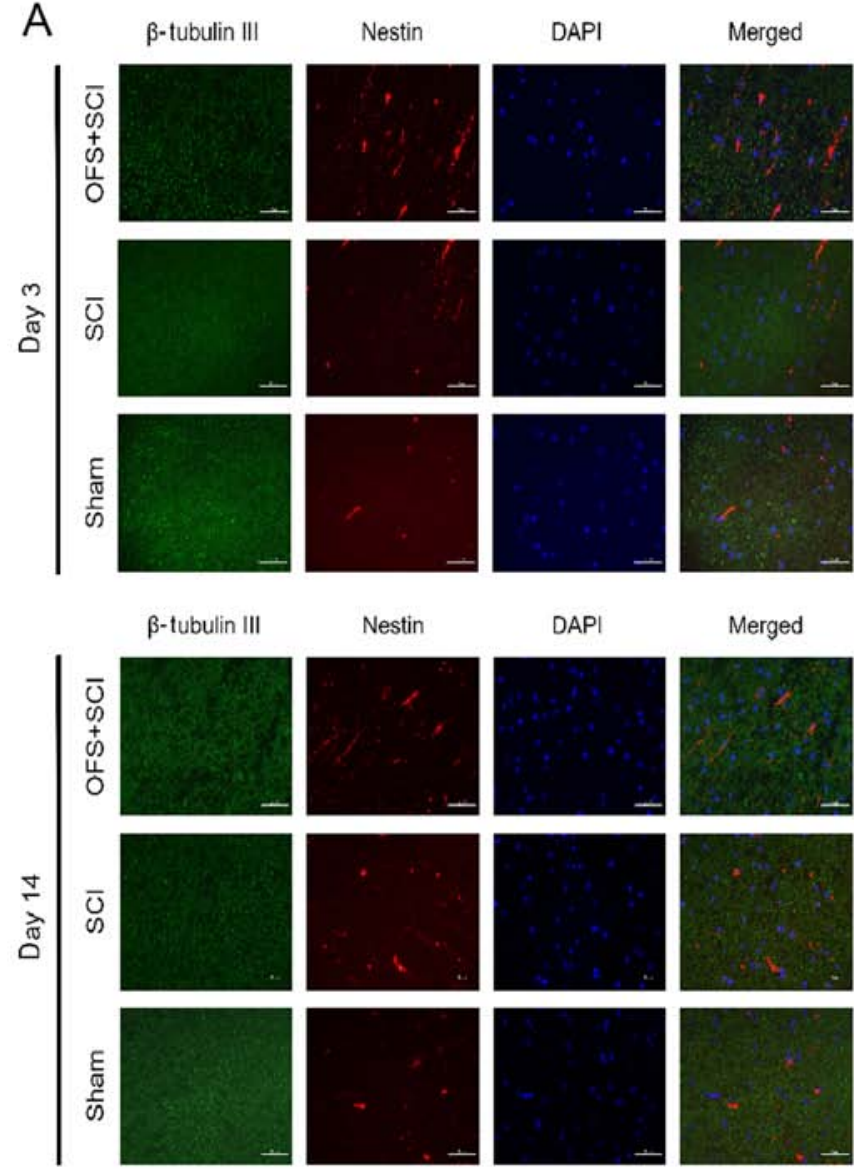

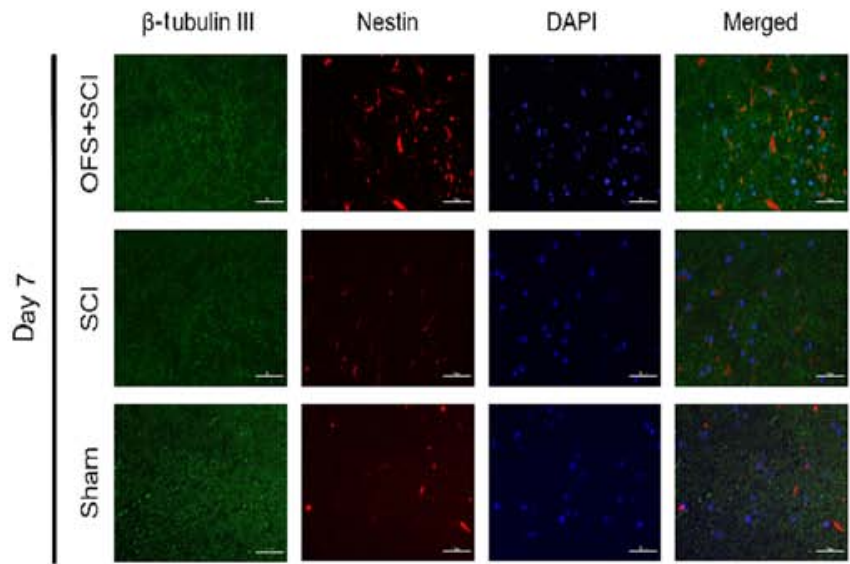

B

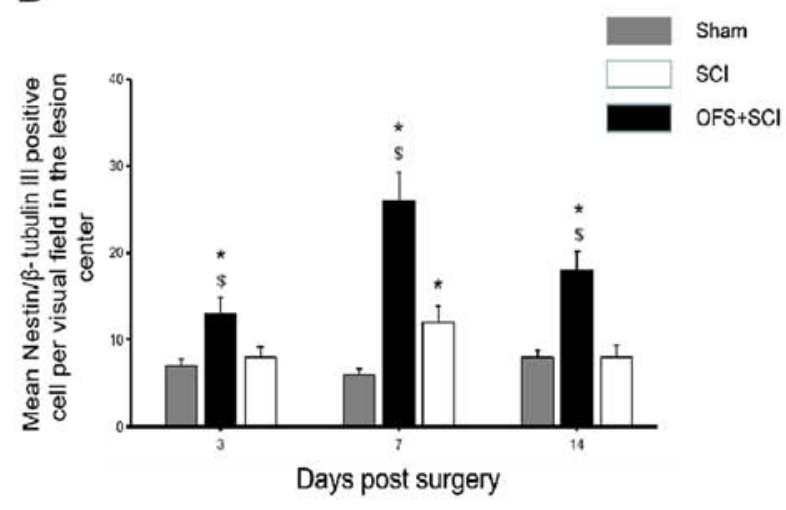

Figure 2. OFS treatment increases the differentiation of endogenous neural stem cells into neurons. (A) Immunofluorescence double-staining was used to identify the expression of Nestin and $\beta$-tubulin III among groups at day 3, 7 and 14 post-surgery. (B) Quantification of Nestin and $\beta$-tubulin III positive cells in the lesion site in each group at each time point. Scale bar, $50 \mu \mathrm{m}$. DAPI staining, blue. Nestin staining, red. $\beta$-tubulin III staining, green. * $<0.05$ vs. Sham group. ${ }^{\$} \mathrm{P}<0.05$ vs. SCI group. Sham, controls that underwent laminectomy only; SCI, group that underwent laminectomy and spinal cord injury; OFS + SCI, group that underwent laminectomy, spinal cord injury and OFS; OFS, oscillating field stimulation.

primary and secondary antibodies combined on the tissue. Subsequently, sections were incubated with the second primary antibody against Nestin (1:100; cat. no. ab6142; Abcam) at $4^{\circ} \mathrm{C}$ in Quickblock primary antibody dilution buffer overnight and stained with Cy3-conjugated goat anti-mouse IgG (1:200; cat. no. GB21301; Servicebio Technology, Co., Ltd.) for $1 \mathrm{~h}$ at room temperature. Finally, nuclei were counterstained with DAPI (cat. no. G1012; Servicebio Technology, Co., Ltd.) for $10 \mathrm{~min}$ at room temperature. Images were acquired using a fluorescent microscope (Eclipse C1; Nikon Corporation) at $\mathrm{x} 400$ magnification. Each group had 36 sections, and 3 visual fields were randomly selected from each section and quantified using Image-Pro Plus software (version 6.0; Media Cybernetics, Inc.).

Hematoxylin and eosin $(H \& E)$ staining. Rats from all experimental groups were sacrificed at 14 days after surgery and a 1-cm segment of SC section centered on the site of injury was removed. Tissues were immediately fixed in $4 \%$ paraformaldehyde at room temperature for $24 \mathrm{~h}$, followed by paraffin embedding. Tissues from all groups were cut into $5-\mu \mathrm{m}$ thick transverse sections for H\&E staining as follows: Sections were stained with Hematoxylin (cat. no. H8070; Beijing Solarbio Science \& Technology Co., Ltd.) at room temperature for
$5 \mathrm{~min}$ and washed with distilled water for $5 \mathrm{~min}$. Then, tissue sections were immersed in $1 \%$ hydrochloric acid alcohol at room temperature for $5 \mathrm{sec}$ and washed with distilled water for $3 \mathrm{~min}$. Each section was immersed in $0.5 \%$ ammonia (cat. no. G1821; Beijing Solarbio Science \& Technology Co., Ltd.) at room temperature for $10 \mathrm{~min}$ and washed with distilled water for $5 \mathrm{~min}$. Subsequently, sections were stained with Eosin Y solution (cat. no. G1100; Beijing Solarbio Science \& Technology Co., Ltd.) at room temperature for $3 \mathrm{~min}$. From each group 12 sections were stained and observed using a fluorescent microscope (Eclipse E100; Nikon Corporation) at x100 magnification for morphological changes (3 visual fields were randomly selected from each section).

Nissl staining. Following sacrifice, a 1-cm SC section centered on the injury site was removed at 14 days postsurgery, immediately fixed in $4 \%$ paraformaldehyde at room temperature for $24 \mathrm{~h}$, embedded in paraffin and cut into $5-\mu \mathrm{m}$ thick transverse sections. From each group 12 sections were stained. Nissl staining was performed according to the manufacturer's protocol (cat. no. G1434; Beijing Solarbio Science \& Technology Co., Ltd.) for $30 \mathrm{~min}$ at room temperature. Sections were observed under a fluorescent microscope (Eclipse E100; Nikon Corporation) at x100 magnification to 

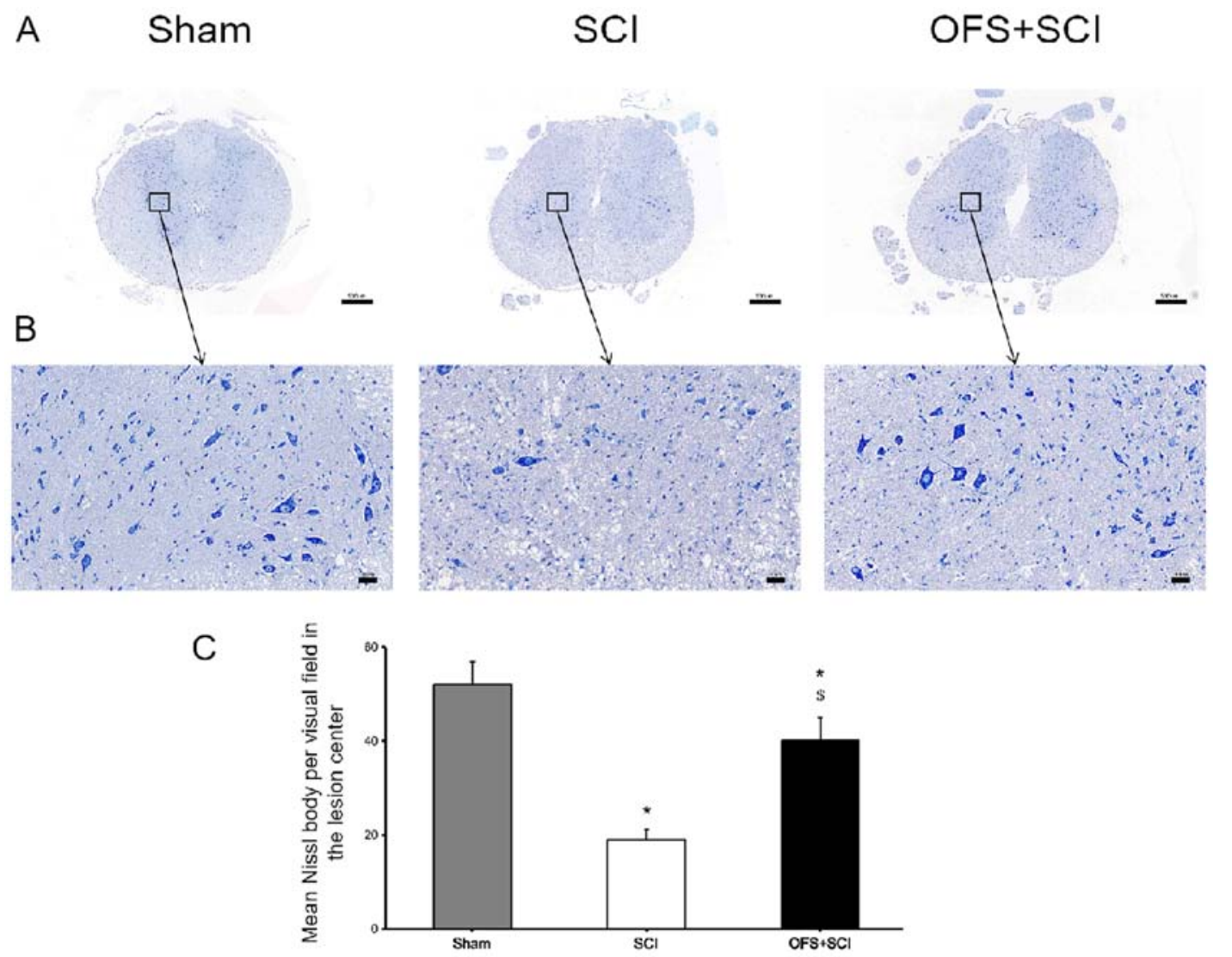

Figure 3. OFS treatment increases the viability of neurons in injured spinal cord. (A and B) Nissl staining was performed 14 days post-surgery. Sham group in the left column, SCI group in the middle and OFS group in the right column. (C) Quantification of Nissl bodies in each group at the lesion site. Scale bar, $500 \mu \mathrm{m}$ in A and $50 \mu \mathrm{m}$ in B. One-way ANOVA followed by Tukey's post hoc test. " $\mathrm{P}<0.05$ vs. Sham group. ${ }^{\$} \mathrm{P}<0.05$ vs. SCI group. OFS, oscillating field stimulation; Sham, controls that underwent laminectomy only; SCI, group that underwent laminectomy and spinal cord injury; SCI + OFS, group that underwent laminectomy, spinal cord injury and OFS.

evaluate morphological changes (3 visual fields were randomly selected from each section).

Uranium-lead staining and transmission electron microscopy (TEM). Following sacrifice, $1-\mathrm{cm}$ SC sections centered on the site of injury were removed at 14 days post-surgery and immediately fixed in $2.5 \%$ glutaraldehyde at room temperature for $12 \mathrm{~h}$, followed by immersion in $1 \%$ osmium acid at room temperature for $2 \mathrm{~h}$. Following dehydration, tissues were embedded in pure epoxy resin and cut into 70-nm thick sections using an ultra-thin slicer (UC-7; Leica Microsystems, Inc.). Finally, sections were stained with uranium and lead at room temperature for $30 \mathrm{~min}$, and observed under a TEM (JEM1400; JEOL, Ltd.) to assess myelin sheath morphology. There were 12 sections for each group and 3 visual fields were randomly selected from each section.

Statistical analysis. Data are presented as mean \pm standard error of mean (SEM) of 3 independent experiments. Multiple group means were compared using two-way ANOVA with or without repeated measures or one-way ANOVA all of which were followed by Tukey's post hoc tests for multiple pair-wise comparisons. Pairs of means were compared using the Student's t-test. SPSS software (version 19.0; SPSS Inc.) was used for all statistical analyses. $\mathrm{P}<0.05$ was considered to indicate a statistically significant difference.

\section{Results}

OFS treatment improves functional recovery following SCI. Hind limb movement and coordination were evaluated using BBB scores to evaluate the efficacy of OFS for SCI treatment. In the Sham group, there was no significant difference in $\mathrm{BBB}$ score at each time point $(\mathrm{P}>0.05)$. Rats receiving OFS following SCI demonstrated markedly higher scores compared with the SCI group rats starting from the 3rd week post-surgery $(\mathrm{P}<0.05$; Fig. 1$)$.

OFS promotes the differentiation of endogenous NSCs into neurons. IF double labeling for $\beta$-tubulin III and the neural stem/progenitor cell marker Nestin was performed at days 3, 7 and 14 post-surgery to investigate whether OFS regulates the differentiation of endogenous NSCs into neurons. There were more Nestin and $\beta$-tubulin III positive cells in the SC sections from the SCI + OFS group compared with the SCI and Sham groups at each measurement time point (Fig. 2A), with the Nestin and $\beta$-tubulin III positive cell number peaking in the SCI + OFS group on the 7th day post-surgery compared with the SCI group ( $\mathrm{P}<0.05$; Fig. 2B).

Nissl and H\&E staining were also performed at day 14 postsurgery to assess the level of tissue pathology. The SCI group exhibited fewer Nissl bodies compared with the SCI + OFS and Sham groups $(\mathrm{P}<0.05$; Fig. 3). In addition, OFS treatment 

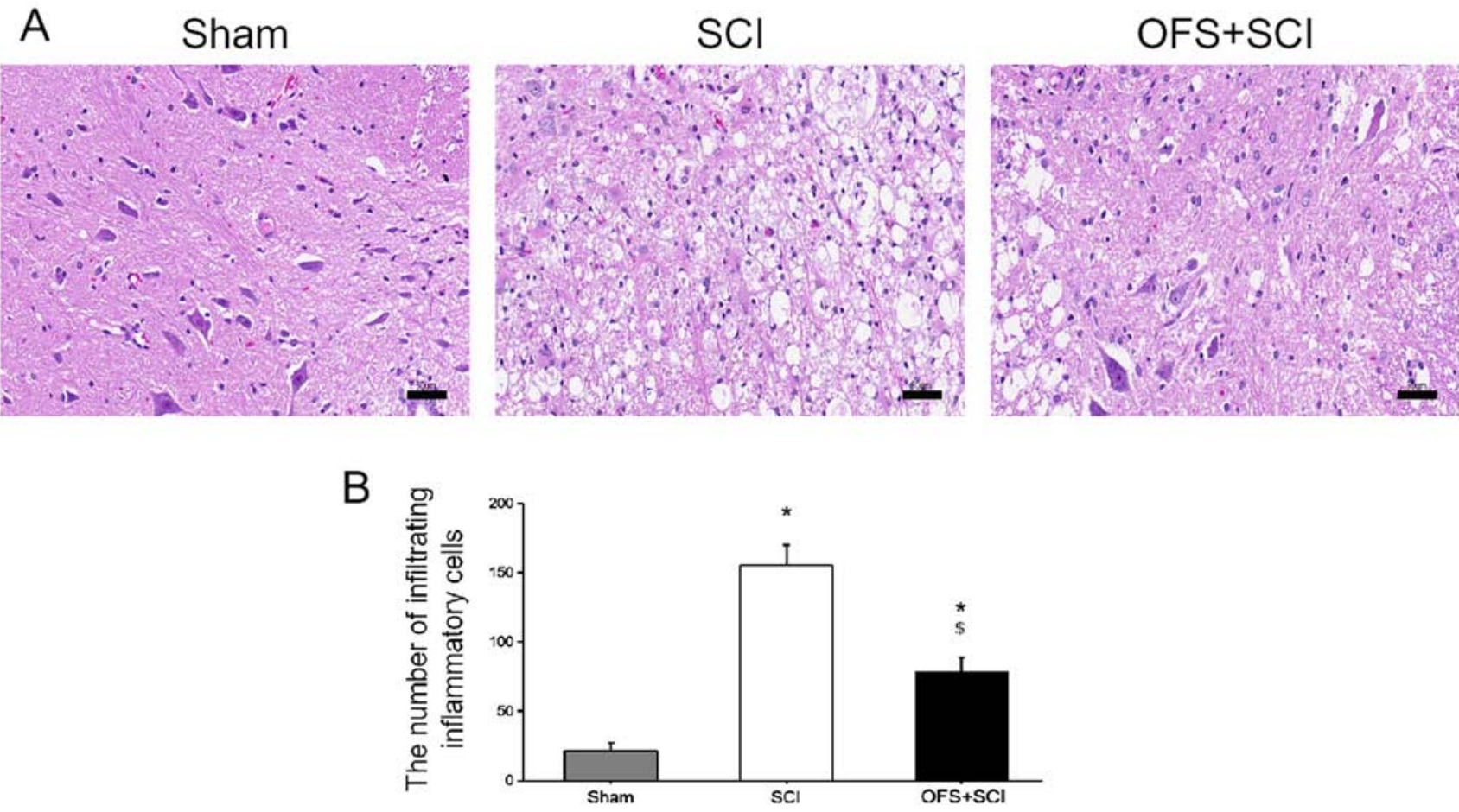

Figure 4. OFS treatment reduced the inflammatory cell infiltration in injured spinal cord. (A) H\&E staining was performed 14 days after surgery. Sham group in the left column, SCI group in the middle, and OFS group in the right column. (B) Quantification of infiltrating inflammatory cells in each group in the lesion site. Scale bar, $50 \mu \mathrm{m}$. One-way ANOVA followed by Tukey's post hoc test. ${ }^{*} \mathrm{P}<0.05$ vs. Sham group. ${ }^{\$} \mathrm{P}<0.05$ vs. SCI group. OFS, oscillating field stimulation; Sham, controls that underwent laminectomy only; SCI, group that underwent laminectomy and spinal cord injury; SCI + OFS, group that underwent laminectomy, spinal cord injury and OFS.

reduced inflammatory cell infiltration compared with the SCI group $(\mathrm{P}<0.05$; Fig. 4).

OFS promotes the differentiation of endogenous NSCs into oligodendrocytes. Double IF staining was performed for Nestin and the oligodendrocyte precursor cell (OPC) marker NG2 at days 3, 7 and 14 post-surgery to examine the effects of OFS on NSC differentiation into oligodendrocytes (Fig. 5A). IF staining revealed a significantly greater number of Nestin and NG2-positive cells in the SCI + OFS group compared with the SCI group at all measurement points $(\mathrm{P}<0.05$; Fig. $5 \mathrm{~B})$, with cell number peaking on the 7 th day post-surgery.

Myelin sheath morphology was examined using TEM to assess the effects of OFS on remyelination following SCI. Compared with the SCI group, the Sham and SCI + OFS group exhibited a significantly greater number of myelin sheaths and thicker sheaths at 14 days post-surgery ( $\mathrm{P}<0.05$; Fig. 6).

\section{Discussion}

Stem cell transplants have demonstrated promising results for the treatment of SCI (17). However, cell rejection and poor in vivo survival have limited the success of this strategy $(18,19)$. Recent studies have shown that endogenous NSCs can improve the self-repair capacity of the SC, indicating that endogenous NSCs may be potential therapeutic targets for SCI treatment $(20,21)$. The differentiation of NSCs depends primarily on their microenvironment following SCI, which is influenced by factors such as edema, ischemia and activation of inflammatory response, and is also an important factor affecting damage repair $(22,23)$. Therefore, regulation of directional differentiation of endogenous NSCs may be the key to SCI repair.

Wnt signaling is an important regulator of NSC proliferation, self-renewal and differentiation in the central nervous system (CNS) $(24,25)$. Various Wnt factors directly regulate the proliferation and differentiation of endogenous NSC (12). Previous studies have reported that Wnt1, Wnt2 and Wnt3a expression results in NSC proliferation in the CNS $(26,27)$. Furthermore, Wnt3a expression directs the differentiation of mouse embryonic stem cells into dorsal interneurons (28) and Wnt7a expression promotes neuronal differentiation of NSCs in mice (29). Additionally, it has been revealed that electric field stimulation alters Wnt protein expression and Wnt signaling, thus regulating the differentiation of NSCs and affecting nerve regeneration and SCI repair. OFS, as a neuroelectric technology, is an emerging neurostimulation modality that has shown promise for facilitating repair after SCI $(10,14)$. The present study also demonstrated that OFS regulated the differentiation of endogenous NSCs following SCI. However, the potential mechanism needs further study.

To the best of our knowledge, the current study was the first to identify that targeted OFS to the SCI site promotes the differentiation of endogenous NSCs into neurons and oligodendrocytes and accelerates recovery of hind limb function. The BBB scores revealed substantially faster and improved locomotor improvement in the SCI + OFS group compared with the SCI group with no apparent adverse effects on survival of rats, indicating that OFS is a feasible strategy for the treatment of SCI. 
A

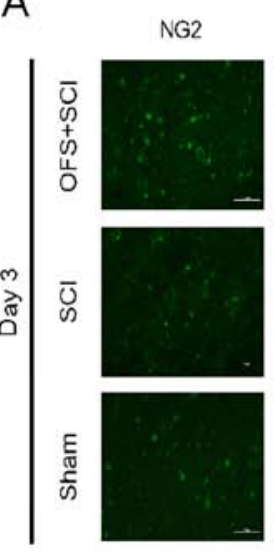

NG2

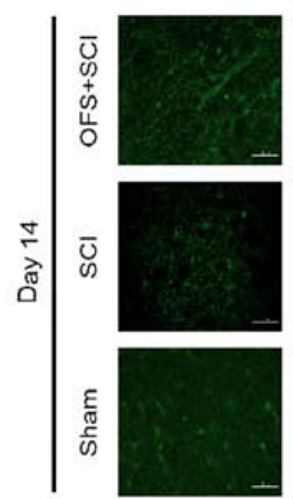

Nestin
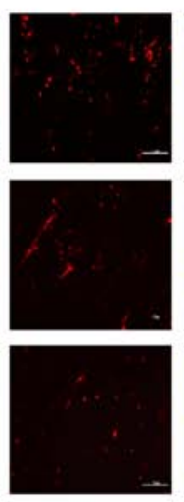

Nestin
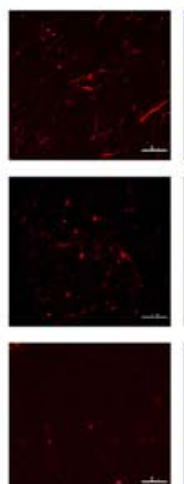

DAPI
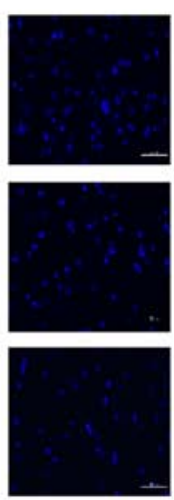

DAPI
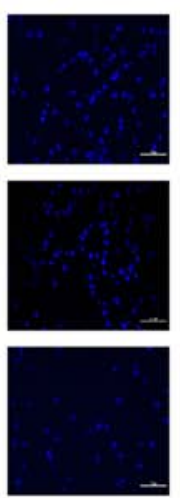

Merged
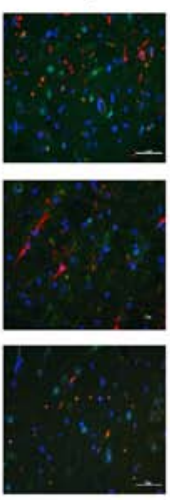

Merged
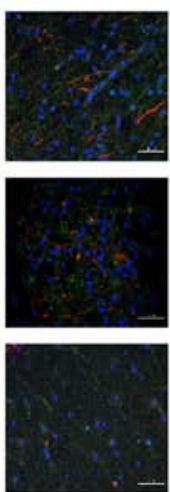

NG2
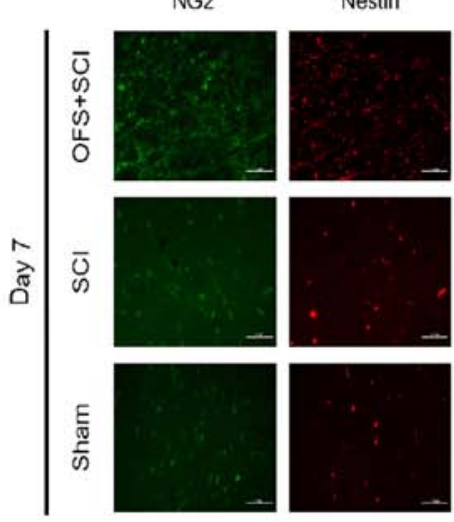

$\mathrm{B}$

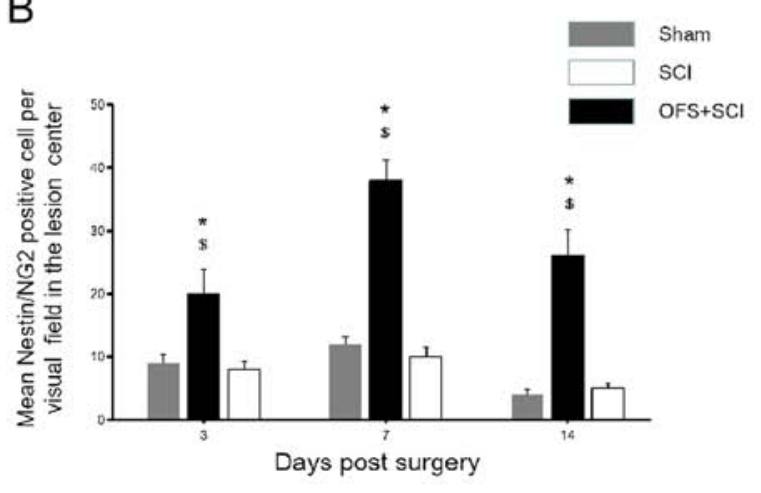

Figure 5. OFS treatment improves the differentiation of endogenous NSCs into oligodendrocytes. (A) Immunofluorescence double-staining was performed to identify the expression of Nestin and NG2 among groups at day 3, 7 and 14 post-surgery. (B) Quantification of Nestin and NG2 positive cells at the lesion site in each group at respective time points. Scale bar, $50 \mu \mathrm{m}$. DAPI staining, blue. Nestin staining, red. NG2 staining, green. ${ }^{*} \mathrm{P}<0.05 \mathrm{vs}$. Sham group. ${ }^{\$} \mathrm{P}<0.05 \mathrm{vs}$. SCI group. NG2, neuron-glial antigen 2; Sham, controls that underwent laminectomy only; SCI, group that underwent laminectomy and spinal cord injury; SCI + OFS, group that underwent laminectomy, spinal cord injury and OFS; OFS, oscillating field stimulation.

A

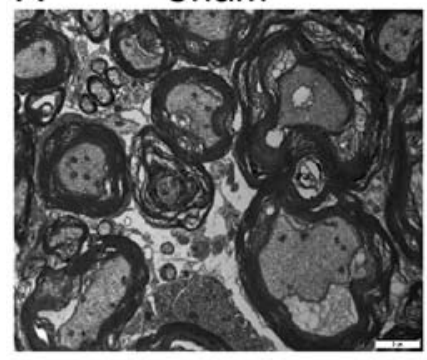

B

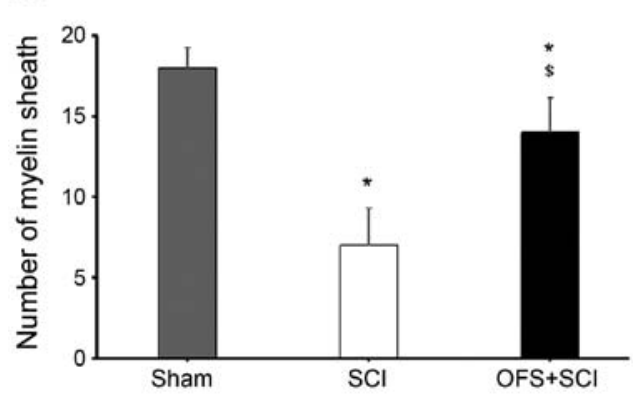

$\mathrm{SCl}$

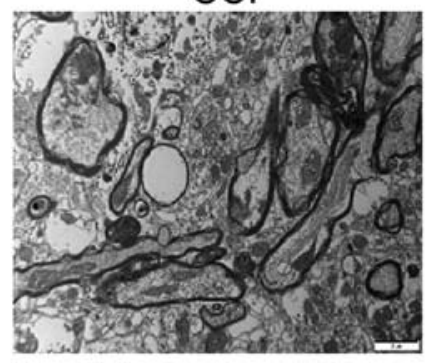

C

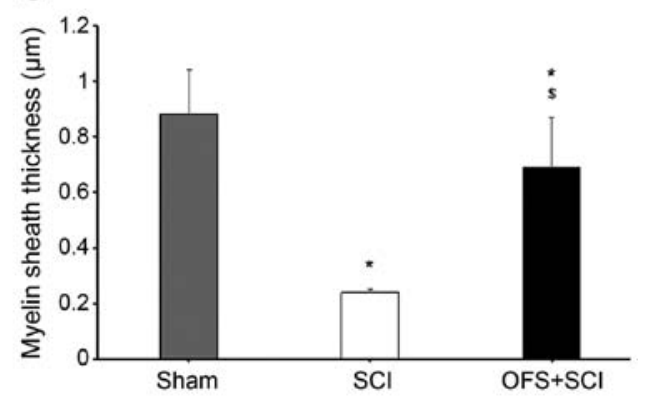

Figure 6. OFS treatment promotes remyelination of nerve fibers in the lesion site. (A) Uranium-lead staining by transmission electron microscopy was used to observe the morphology of myelin sheath at 14 days post-surgery. Sham group in the left column, SCI group in the middle and OFS group in the right column. (B) Quantification of the number of myelin sheaths in each group. (C) Quantification of myelin sheath thickness in each group. One-way ANOVA followed by Tukey's post hoc test. Scale bar, $2 \mu \mathrm{m}$. " $\mathrm{P}<0.05$ vs. Sham group.. ${ }^{\$} \mathrm{P}<0.05$ vs. SCI group. OFS, oscillating field stimulation; Sham, controls that underwent laminectomy only; SCI, group that underwent laminectomy and spinal cord injury; OFS + Sci, group that underwent laminectomy, spinal cord injury and OFS. 
$\beta$-tubulin is one of the main components of the cytoskeleton and the class III isoform is expressed exclusively in neurons, where it contributes to morphological development and axon orientation (30). NSCs promote SC repair by enhancing neuronal viability, reducing neuroinflammation and replenishing cells lost to apoptosis (31). Although axonal repair or regeneration have been the main focus of SCI research, regeneration of oligodendrocytes and remyelination are also critical for recovery of neurological function after SCI (32). The present results suggested that OFS enhanced the production of NSC-derived oligodendrocytes and neurons in the early post-SCI phase as shown by Nestin and NG2 double labeling and Nestin and $\beta$-tubulin III double labeling, respectively. Nissl staining identified more neurons in SCI rats receiving OFS. Additionally, H\&E staining demonstrated less infiltration of inflammatory cells in the OFS group compared with SCI group. Collectively, these results indicated that OFS therapy promoted the differentiation of endogenous NSCs into viable neurons.

Previous studies have identified OPCs in the mature mammalian CNS that contribute to the repair of nerve fiber demyelination (33-35). It has also been shown that OFS can enhance the differentiation of OPCs into oligodendrocytes and promote remyelination following SCI in rats (14). In the current study, it was found that Nestin and NG2-positive cell numbers at the lesion site and Nestin and NG2 expression levels were significantly increased as early as 3 days following SCI in the OFS group. Uranium-lead staining and TEM directly demonstrated that OFS increased the number of myelinated fibers and myelin sheath thickness. Thus, OFS therapy improved the differentiation of endogenous NSCs into oligodendrocytes, thereby promoting remyelination of nerve fibers following $\mathrm{SCI}$ in rats.

A total of $19 \mathrm{Wnt}$ proteins have been discovered in mammals and some of these are expressed at various stages of CNS development (29). In the process of treating SCI, the degree of interaction between OFS and the Wnt signaling pathway, as well as the specific Wnt proteins involved in repair remain unknown. Therefore, these factors require further investigation.

In conclusion, OFS promotes NSC differentiation into neurons and oligodendrocytes following SCI in rats, thereby accelerating axonal regeneration, remyelination and neurological recovery. Therefore, it was speculated that OFS may be a safe and effective strategy for the treatment of SCI, either alone or in conjunction with NSC transplant.

\section{Acknowledgements}

Not applicable.

\section{Funding}

The current study was supported by the National NaturalScience Foundation of China (grant no. 81471273 and 81671204), theExcellent Young Talents Support Program in Universities(grant no. gxyqZD2017032) and the Natural science research projects in Anhui Universities (grant no. KJ2020ZD23).

\section{Availability of data and materials}

The datasets used and/or analyzed during the current study are available from the corresponding author on reasonable request.

\section{Authors' contributions}

CF and JQ designed this research and wrote the manuscript; CF and JS performed the experiments; LW and FG collected and analyzed the data. All authors read and approved the final manuscript.

\section{Ethics approval and consent to participate}

All surgical procedures and experiments were performed in accordance with the National Institutes of Health Guide for the Care and Use of Laboratory Animals (NIH Publications no. 80-23; revised 1996) and were approved by the Animal Ethics Committee of Anhui Medical University (approval reference no. LLSC20190736). All applicable international, national and/or institutional guidelines for the care and use of animals were followed.

\section{Patient consent for publication}

Not applicable.

\section{Competing interests}

The authors declare that they have no competing interests.

\section{References}

1. Wyndaele M and Wyndaele JJ: Incidence, prevalence and epidemiology of spinal cord injury: What learns a worldwide literature survey? Spinal Cord 44: 523-529, 2006.

2. Singh A, Tetreault L, Kalsi-Ryan S, Nouri A and Fehlings MG: Global prevalence and incidence of traumatic spinal cord injury. Clin Epidemiol 6: 309-331, 2014.

3. Middleton JW, Dayton A, Walsh J, Rutkowski SB, Leong G and Duong S: Life expectancy after spinal cord injury: A 50-year study. Spinal Cord 50: 803-811, 2012.

4. Devivo MJ: Epidemiology of traumatic spinal cord injury: Trends and future implications. Spinal Cord 50: 365-372, 2012.

5. Dyck SM and Karimi-Abdolrezaee S: Chondroitin sulfate proteoglycans: Key modulators in the developing and pathologic central nervous system. Exp Neurol 269: 169-187, 2015.

6. Sabelström H, Stenudd M and Frisén J: Neural stem cells in the adult spinal cord. Exp Neurol 260: 44-49, 2014.

7. Grégoire CA, Goldenstein BL, Floriddia EM, Barnabé-Heider F and Fernandes KJ: Endogenous neural stem cell responses to stroke and spinal cord injury. Glia 63: 1469-1482, 2015.

8. Stenudd M, Sabelström H and Frisén J: Role of endogenous neural stem cells in spinal cord injury and repair. JAMA Neurol 72: 235-237, 2015.

9. Taveggia C,Feltri ML and Wrabetz L: Signals to promote myelin formation and repair. Nat Rev Neurol 6: 276-287, 2010.

10. Shapiro S, Borgens R, Pascuzzi R, Roos K, Groff M, Purvines S, Rodgers RB, Hagy S and Nelson P: Oscillating field stimulation for complete spinal cord injury in humans: A phase 1 trial. J Neurosurg Spine 2: 3-10, 2005.

11. Wu Y, Collier L, Qin W, Creasey G, Bauman WA, Jarvis J and Cardozo C: Electrical stimulation modulates Wnt signaling and regulates genes for the motor endplate and calcium binding in muscle of rats with spinal cord transection. BMC Neurosci 14: 81, 2013.

12. Piccin D and Morshead CM: Wnt signaling regulates symmetry of division of neural stem cells in the adult brain and in response to injury. Stem Cells 29: 528-538, 2011.

13. Li Z, Yao F, Cheng L, Cheng W, Qi L, Yu S, Zhang L, Zha X and Jing J: Low frequency pulsed electromagnetic field promotes the recovery of neurological function after spinal cord injury in rats. J Orthop Res 37: 449-456, 2019.

14. Jing JH, Qian J, Zhu N, Chou WB and Huang XJ: Improved differentiation of oligodendrocyte precursor cells and neurological function after spinal cord injury in rats by oscillating field stimulation. Neuroscience 303: 346-351, 2015. 
15. Cho SR, Kim YR, Kang HS, Yim SH, Park CI, Min YH, Lee BH, Shin JC and Lim JB: Functional recovery after the transplantation of neurally differentiated mesenchymal stem cells derived from bone marrow in a rat model of spinal cord injury. Cell Transplant 25: 1423, 2016.

16. Basso DM, Beattie MS, Bresnahan JC, Anderson DK, Faden AI, Gruner JA, Holford TR, Hsu CY, Noble LJ, Nockels R, et al: MASCIS evaluation of open field locomotor scores: Effects of experience and teamwork on reliability. Multicenter Animal Spinal Cord Injury Study. J Neurotrauma 13: 343-359, 1996.

17. Cofano F, Boido M, Monticelli M, Zenga F, Ducati A, Vercelli A and Garbossa D: Mesenchymal stem cells for spinal cord injury: current options, limitations, and future of cell therapy. Int J Mol Sci 20: 2698, 2019.

18. Galderisi U, Peluso G, Di Bernardo G, Calarco A, D'Apolito M, Petillo O, Cipollaro M, Fusco FR and Melone MA: Efficient cultivation of neural stem cells with controlled delivery of FGF-2. Stem Cell Res (Amst) 10: 85-94, 2013.

19. Hernández J, Torres-Espín A and Navarro X: Adult stem cell transplants for spinal cord injury repair: Current state in preclinical research. Curr Stem Cell Res Ther 6: 273-287, 2011.

20. Liu S and Chen Z: Employing Endogenous NSCs to Promote Recovery of Spinal Cord Injury. Stem Cells Int 2019: 1958631, 2019.

21. Yu JH, Seo J-H, Lee JY, Lee M-Y and Cho S-R: Induction of neurorestoration from endogenous stem cells. Cell Transplant 25 863-882, 2016

22. Sandner B, Prang P, Rivera FJ, Aigner L, Blesch A and Weidner N: Neural stem cells for spinal cord repair. Cell Tissue Res 349: 349-362, 2012.

23. Schwab ME: Repairing the injured spinal cord. Science 295: 1029-1031, 2002.

24. Willert K, Brown JD, Danenberg E, Duncan AW, Weissman IL Reya T, Yates JR III and Nusse R: Wnt proteins are lipid-modified and can act as stem cell growth factors. Nature 423: 448-452, 2003.

25. David MD, Cantí C and Herreros J: Wnt-3a and Wnt-3 differently stimulate proliferation and neurogenesis of spinal neural precursors and promote neurite outgrowth by canonical signaling. J Neurosci Res 88: 3011-3023, 2010.
26. Sousa KM, Villaescusa JC, Cajanek L, Ondr JK, CasteloBranco G, Hofstra W, Bryja V, Palmberg C, Bergman T, Wainwright $\mathrm{B}$, et al: Wnt2 regulates progenitor proliferation in the developing ventral midbrain. J Biol Chem 285: 7246-7253, 2010.

27. Megason SG and McMahon AP: A mitogen gradient of dorsal midline Wnts organizes growth in the CNS. Development 129: 2087-2098, 2002.

28. Murashov AK, Pak ES, Hendricks WA, Owensby JP, Sierpinski PL, Tatko LM and Fletcher PL: Directed differentiation of embryonic stem cells into dorsal interneurons. FASEB J 19: 252-254, 2005.

29. Hirabayashi Y, Itoh Y, Tabata H, Nakajima K, Akiyama T, Masuyama $\mathrm{N}$ and Gotoh $\mathrm{Y}$ : The Wnt/beta-catenin pathway directs neuronal differentiation of cortical neural precursor cells. Development 131: 2791-2801, 2004.

30. Binarová P and Tuszynski J: Tubulin: structure, functions and roles in disease. Cells 8: 1294, 2019.

31. Lu P, Wang Y, Graham L, McHale K, Gao M, Wu D, Brock J, Blesch A, Rosenzweig ES, Havton LA, et al: Long-distance growth and connectivity of neural stem cells after severe spinal cord injury. Cell 150: 1264-1273, 2012.

32. Hesp ZC, Goldstein EZ, Miranda CJ, Kaspar BK and McTigue DM: Chronic oligodendrogenesis and remyelination after spinal cord injury in mice and rats. J Neurosci 35: 1274-1290, 2015.

33. Kang SH, Fukaya M, Yang JK, Rothstein JD and Bergles DE: $\mathrm{NG}^{+} \mathrm{CNS}$ glial progenitors remain committed to the oligodendrocyte lineage in postnatal life and following neurodegeneration. Neuron 68: 668-681, 2010.

34. Guo YE, Suo N, Cui X, Yuan Q and Xie X: Vitamin C promotes oligodendrocytes generation and remyelination. Glia 66: 1302-1316, 2018.

35. Skaper SD: Oligodendrocyte precursor cells as a therapeutic target for demyelinating diseases. Prog Brain Res 245: 119-144, 2019.

(†) $\ominus$ This work is licensed under a Creative Commons Attribution-NonCommercial-NoDerivatives 4.0 International (CC BY-NC-ND 4.0) License. 